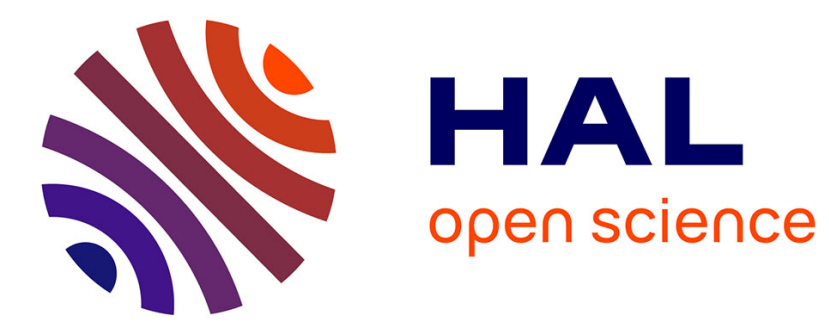

\title{
Les relations sexuelles des seniors vivant avec le VIH
}

\author{
Maks Banens
}

\section{To cite this version:}

Maks Banens. Les relations sexuelles des seniors vivant avec le VIH. Sexologies, 2016, 10.1016/J.sexol.2016.06.003 . halshs-01368978

\section{HAL Id: halshs-01368978 https://shs.hal.science/halshs-01368978}

Submitted on 22 Sep 2016

HAL is a multi-disciplinary open access archive for the deposit and dissemination of scientific research documents, whether they are published or not. The documents may come from teaching and research institutions in France or abroad, or from public or private research centers.
L'archive ouverte pluridisciplinaire HAL, est destinée au dépôt et à la diffusion de documents scientifiques de niveau recherche, publiés ou non, émanant des établissements d'enseignement et de recherche français ou étrangers, des laboratoires publics ou privés. 
Sexologies (2016) 25, 122-127

\title{
LES RELATIONS SEXUELLES DES SENIORS VIVANT AVEC LE VIH
}

\author{
M. Banens
}

\author{
Centre Max Weber - CNRS, Université Lyon \\ Maks.Banens@univ-lyon2.fr
}

L'article interroge l'activité sexuelle des séniors vivant avec le VIH ainsi que son contexte conjugal et social. L'activité sexuelle des séniors séropositifs est l'une des préoccupations suscitées par le rapide vieillissement de la population séropositive. Elle est considérée comme l'une des dimensions de bien-être et d'intégration sociale. Plus ou moins étroitement liée à la conjugalité, elle renseigne également sur l'éventuelle prise en charge des séniors séropositifs quand le besoin se fera sentir.

Une enquête a été menée auprès de 125 séropositifs suivis dans les hôpitaux du COREVIH Vallée du Rhône (co-financeur de l'enquête), dont 80 par questionnaire et 45 par entretien approfondi. Au total, 80 séniors (50 ans et plus) ont été étudiés et comparés à 45 séropositifs plus jeunes. L'échantillon est constitué de façon aléatoire selon les rendez-vous à l'hôpital, mais le taux de refus est trop élevé pour considérer l'échantillon comme représentatif. Il a permis néanmoins de décrire une grande variété de situations et de repérer des régularités à l'intérieur des différentes catégories de séniors séropositifs.

Ainsi, hommes et femmes hétérosexuels, hommes homosexuels et bisexuels constituent quatre configurations contrastées. Les hommes hétérosexuels ont tendance à continuer de vivre dans leur environnement familial (femme, enfants, parfois petits-enfants), mais sur un mode conflictuel et sans activité sexuelle. Les femmes hétérosexuelles vivent souvent seules - comme au moment de leur contamination - mais en bons termes avec leurs enfants. Les hommes homosexuels vivent le plus souvent en couple séroconcordant, couple post-dépistage, affectif, harmonieux, mais peu sexuel. Les hommes bisexuels, enfin, vivent souvent seuls, sans activité sexuelle, en situation conflictuelle avec enfants et ex-compagnes. Ils semblent les plus isolés socialement et les plus fragiles psychologiquement. 


\section{Introduction}

La population séropositive vieillit. Près d'une personne séropositive sur deux a plus de cinquante ans. Elles étaient $7 \%$ en 1995, $20 \%$ en 2003, $41 \%$ en 2011 (Lert, et al, 2013 ; UNAIDS, 2013). Le vieillissement touche les séropositifs en tant que groupe, pour des raisons qui ne sont pas discutées ici, mais aussi les personnes individuellement. L'hypothèse du vieillissement physiologique accéléré, sous les effets combinés du virus et des traitements anti-rétroviraux, semble désormais confirmée (Morlat, 2014, Iwugi, 2013). Face à ce double vieillissement, une inquiétude a vu le jour sur les conditions de vie des seniors séropositifs, et notamment sur la présence ou absence d'un environnement social susceptible de prendre soin d'eux quand le besoin s'en fera sentir (ANRS, 2009; Groupe SOS, 2013). Dans ce cadre, nous avons mené une enquête sur les seniors suivis dans les hôpitaux du COREVIH Vallée du Rhône. L'enquête est présentée ci-dessous. Cet article traite de l'un des aspects du fonctionnement social des seniors séropositifs, leurs relations sexuelles.

De nombreuses recherches ont démontré les interactions entre activité sexuelle, satisfaction conjugale et bien être psychologique des seniors (Trudel et al, 2013 ; Trudel et al, 2014 ; Widmer et al, 2014). Même si le sens des interactions n'est pas bien connu, l'activité sexuelle semble un indicateur robuste pour le bien être psychologique et la satisfaction conjugale. Il reste à vérifier, toutefois, que cela s'applique également aux différentes sous-populations séropositives.

L'activité sexuelle des seniors se situe sur la croisée de deux tendances. D'une part, elle diminue avec l'âge, aujourd'hui comme hier (Kontula et al, 2009). D'autre part, à âge égal, elle augmente fortement depuis plusieurs décennies (Le Van et al, 2008). Cependant, les seniors séropositfs ne sont pas seulement seniors, ils sont également porteurs d'une maladie chronique, dont on connaît les effets négatifs sur l'activité sexuelle (Colson, 2016 ; Levinson, 2008). On s'attend donc à trouver une plus faible activité sexuelle chez les seniors séropositifs, peut-être corrélée à moins de bien être, moins de conjugalité et moins de satisfaction conjugale.

Lovejoy et al. (2008) ont effectivement constaté une très faible activité sexuelle parmi les 290 seniors vivant avec le VIH, principalement à New York City: seuls $38 \%$ étaient sexuellement actifs au cours des trois derniers mois. Les hommes ayant des rapports sexuels 
avec des hommes (HSH) se trouvaient proches de la moyenne (36\%), les femmes bien endessous $(21 \%)$, les hommes hétérosexuels très au-dessus (72\%). L'étude confirmait bien la dichotomie entre HSH aisés et blancs d'un côté, hommes et femmes hétérosexuels précaires et non-blancs de l'autre (Joyce et al, 2005), mais l'activité sexuelle ne suivait pas cette dichotomie. Elle semblait mieux se maintenir chez les hommes hétérosexuels, malgré leur précarité et problèmes de santé.

De leur côté, Golub et al. (2009) confirment la faible activité sexuelle des seniors vivant avec le VIH, mais n'observent pas de conservation de l'activité sexuelle chez les hommes hétérosexuels.

Pour la France, nous ne disposons pas de données comparables. L'enquête VESPA 2 (Lert et al, 2013) ne fait pas de distinction entre seniors et personnes de moins de cinquante ans. Toutefois, rien ne laisse supposer un écart en faveur des hommes hétérosexuels. Sur l'ensemble des âges, les hommes hétérosexuels sont sexuellement moins actifs que les HSH, mais il n'est pas exclu que la situation soit inversée après l'âge de 50 ans.

C'est précisément l'hypothèse d'Isabelle Wallach (Wallach, 2011, Wallach et al., 2013). Elle constate une chute rapide de l'activité sexuelle des seniors HSH due, selon elle, à l'âgisme plus prononcé à l'intérieur de la communauté gay. Les seniors HSH seraient donc les plus à risque d'isolement social.

Dans le contexte français, l'enquête qualitative de Desesquelles et al. (2013) arrive à une conclusion différente. Pour ces auteurs, les seniors, dans l'ensemble, «vont bien », à l'exception des femmes, qui seraient davantage isolées affectivement et socialement. Leur situation serait symbolisée par la «figure singulière des veuves» dont la « souffrance ne peut trouver d'apaisement [à cause du] sentiment de trahison ». (Desesquelles et al, 2013 : 55) Isolement social, souffrance psychologique et faible activité sexuelle se trouveraient alors, du moins en France, du côté des femmes.

\section{Données et méthodes}

Pour mieux connaître la situation sociale des seniors séropositifs, nous avons mené l'enquête Vieillir avec le VIH auprès des seniors suivis dans les hôpitaux du COREVIH Vallée du Rhône (Banens et al, 2015). L'enquête fut initiée par Rommel Mendès-Leite peu avant sa maladie, puis suivie par lui jusqu'à son décès, une semaine après la sortie du rapport. Au total, 127 personnes ont participé à l'enquête. Sans représenter rigoureusement les seniors de la 
Vallée du Rhône, l'échantillon s'en approche dans la mesure où la demande de participation émanait des services de soin et que les refus furent limités en nombre. Toutefois, le dispositif ne permettant pas de mener plusieurs entretiens à la fois, une partie des répondants ont répondu par questionnaire auto-déclaratif. $\mathrm{Au}$ total, nous avons recueilli 45 entretiens approfondis (durée de 1,5 à 2 heures) et 82 questionnaires : $41 \%$ hommes homosexuels, $11 \%$ hommes bisexuels (11\%), $22 \%$ hommes hétérosexuels et $26 \%$ de femmes hétérosexuelles. Tous les entretiens ont été menés avec des seniors (cinquante ans ou plus). Dans les questionnaires recueillis, 35 sont seniors, les autres, plus jeunes, forment un groupe de comparaison. Les seniors sont souvent jeunes : $80 \%$ ont entre 50 et 65 ans. Cette «jeunesse » est le fait de plus de $95 \%$ des femmes et des hommes homo- et bisexuels. Les hommes hétérosexuels, en revanche, sont sensiblement plus âgés : $42 \%$ ont plus de 65 ans.

\section{Résultats}

Quarante pour cent des seniors, déclarent avoir un partenaire sexuel. Quelques-uns ont une activité sexuelle occasionnelle sans déclarer de partenaire, mais dans l'ensemble, l'activité sexuelle est faible, comparée à celle de la population générale (Bajos et al, 2008), confirmant ainsi Lovejoy et al. (2008) et Wallach (2013). Notons toutefois, que dans notre enquête les hommes hétérosexuels (32\%) se situent en bas du classement, les hommes homosexuels (46\%) en haut du classement, les hommes bisexuels (40\%) et les femmes (40\%) dans une moyenne intermédiaire. Dans toutes les catégories, les sexuellement actifs sont donc minoritaires. Mais la construction, le vécu et les conséquences de cette faible activité varient fortement selon les catégories d'appartenance.

\subsection{Hommes homosexuels}

Chez les hommes homosexuels, le fait de déclarer un partenaire sexuel semble indépendant de l'âge : $48 \%$ avant 50 ans, $46 \%$ après. Mais derrière cette apparente stabilité se cache une importante transformation du cadre de vie. La vie en couple augmente spectaculairement : de $32 \%$ avant 50 ans, elle double presque (56\%) après cet âge. De nombreux hommes vivant seuls se sont donc (re)mis en couple et ce, très majoritairement, avec un autre homme séropositif : la part des couples séroconcordants fait un bond de $13 \%$ avant 50 ans à $71 \%$ après. Troisième particularité, de nombreux conjoints ne sont pas, ou plus, des partenaires sexuels.

Igor (tous les prénoms sont fictifs), 62 ans : «La relation avec l'ami dont je parlais, y'a plus beaucoup de sexe entre nous. » Georges, 76 ans : «Oh j'ai plus de libido. Plus du tout de 
rapports sexuels depuis quatre ou cinq ans. » Henri 54 ans : "C'est la barrière des 50 ans, elle est passée en travers. Plus envie.» Mathieu, 60 ans: "[Vous vivez avec votre compagnon ?] Oui. [Vous avez une activité sexuelle ?] Non. »

Certains ont des partenaires occasionnels ou réguliers en dehors du couple. La pratique du multi-partenariat occasionnel est une caractéristique spécifique des HSH. Selon l'enquête VESPA2 (Lert et al, 2013), $59 \%$ des HSH séropositifs ont eu plus d'un partenaire sexuel au cours des douze derniers mois, contre 11 à $29 \%$ pour les autres groupes. Nous observons ce multi-partenariat occasionnel chez les seniors homosexuels de notre enquête, mais comme phénomène minoritaire. La majorité des hommes vivant en couple sans activité sexuelle conjugale n'ont plus d'activité sexuelle du tout. Ils évoquent souvent la distinction entre sexualité et affection. Henri, 54 ans : "Quand je pratiquais le sexe, c'était pas de l'amour. C'était la possession, de l'exploit physique, du plaisir, mais pas de l'amour. » Igor, 62 ans : «La vie sentimentale, c'est une chose, le sexe, c'est complètement à part. »

La dissociation a souvent comme objectif de valoriser les sentiments affectifs et les témoins font souvent un rapport direct avec l'âge. Raphaël, 57 ans : «Le fait d'avoir stoppé la drague extérieure m'a permis d'avoir un regard différent et de rencontrer cet ami. » Gabriel, 55 ans : «J'ai été ce qu'on appelle un toxico du cul, hein. Pour être honnête, aujourd'hui, d'en être libéré, c'est un soulagement. » Henri, 54 ans : «A partir de 40 ans je me suis dit,... il faut passer à une stabilité, tu as fait le tour. » Joël, 54 ans : «J'ai transposé la sexualité à la sentimentalité [...] Je cherche plus du vrai sentiment. » Et cet affectif, on le recherche de préférence entre séropositifs. Gabriel, 55 ans : «Quand je l'ai quitté [ex-compagnon séronégatif], je me suis dit: plus jamais je m'investirai dans un couple sérodiscordant. » Roger, 52 ans : «J'aurais pas cherché à être avec quelqu'un de séronégatif. »

Si la majorité des seniors séropositifs homosexuels vit en couple - sans que ce couple soit toujours sexuel - une minorité continue de vivre en solitaire. Avant l'âge de 50 ans, ce mode de vie se conjugue bien avec une activité sexuelle. Il en va autrement après. De nombreux seniors, auparavant très actifs, disent vivre presque sans sexualité aujourd'hui. Joël, 54 ans : «Je pourrais me faire moine. » Nicolas, 58 ans : «Pas de partenaire, non, enfin, un rapport sexuel de temps en temps. » Renaud, 61 ans : «Pas de sexualité depuis des années. » Hubert, 53 ans : «Je n'ai plus de vie sexuelle depuis 1997. » L'âgisme constaté par Wallach (2013), revient dans de nombreux témoignages. Henri, 54 ans : «C'est plus la peine de rentrer dans un sauna, vous passez pour une antiquité. » 
La part de ceux déclarant un partenaire sexuel reste stable, mais la fréquence sexuelle semble nettement en baisse, à l'intérieur du couple comme à l'extérieur. Cela ne signifie pas l'isolement social et l'insatisfaction conjugale soient plus présents. Le contraire est vrai. Le repli sur le bonheur domestique, hypothèse émise par Philippe Adam (Adam, 1999), semble effectivement avoir lieu, mais avant tout chez les hommes séropositifs plus âgés, à la recherche d'un entre soi séropositif.

\subsection{Hommes hétérosexuels}

A l'inverse des hommes homosexuels, la déclaration d'un partenaire sexuel s'écroule pour les hommes hétérosexuels : $71 \%$ avant l'âge de 50 ans, $32 \%$ après. Simultanément, la vie de couple se maintient, voire augmente, en passant de $50 \%$ à $58 \%$. A la différence des hommes homosexuels, les hommes hétérosexuels se séparent rarement de leur conjointe après la découverte du VIH, même si, souvent, les relations sexuelles s'arrêtent. Karl, 74 ans : «[Relations sexuelles?] Ah non, c'est fini ça. » Emmanuel, 67 ans : «A partir du moment où on a découvert ce problème [...] elle préférait ne pas avoir de rapports. »

Dans certains cas, l'enchaînement s'est déroulé en sens inverse : les relations sexuelles dans le couple s'étaient arrêtées, ce qui a conduit l'homme à avoir des rapports extraconjugaux. André, 71 ans : «Tant que ça marchait avec ma femme. Mais à partir de 62 ans... j'ai eu l'occasion d'aller... comme ça quoi.» Emmanuel, 67 ans : «Mon épouse se trouvait être extrêmement peu portée sur ce type de ... de rencontre sportive, hein. » Ce scénario semble soutenu par deux autres observations : la part de couples séroconcordants est faible avant l'âge de 50 ans (25\%) comme après 50 ans (27\%), indiquant des rapports sexuels conjugaux peu fréquents entre la contamination et le moment du dépistage. Deuxièmement, les hommes hétérosexuels sont nettement plus âgés que les autres groupes séropositifs, suggérant que la pratique du multi-partenariat ait commencé à un âge plus élevé que chez les hommes homosexuels.

L'âge plus élevé explique certainement une partie de la baisse de l'activité sexuelle. Karl, 74 ans : «Y a longtemps que je peux plus et personne veut me donner quoique ce soit vue que j'ai le cour malade. » Toutefois, la mésentente dans les couples semble être le facteur principal. Karl, 74 ans : «Oui, oui, je suis un salaud, je suis allé coucher ailleurs, c'est tout... Une femme, c'est ça... » Les seuls hommes hétérosexuels déclarant avoir des rapports sexuels avec leur compagne sont ceux qui, séparés de leur conjointe après la découverte du VIH, se sont remis en couple ensuite. Or, ces séparations/remises en couple sont peu fréquentes. 
Dans l'ensemble, les hommes hétérosexuels semblent donc peu actifs sexuellement, à l'extérieur comme à l'intérieur du couple. S'ils vivent aussi fréquemment en couple que les seniors homosexuels, leurs rapports avec femme, enfants et petits-enfants sont beaucoup plus souvent conflictuels, leur activité sexuelle beaucoup plus faible.

\subsection{Hommes bisexuels}

Avant l'âge de 50 ans, aucun des hommes bisexuels de notre enquête n'a un partenaire sexuel et aucun ne vit en couple. Ils déclarent par ailleurs de fréquentes dépressions et une faible confiance en soi. Après 50 ans, ils vivent toujours rarement en couple (20\%), mais déclarent plus souvent des partenaires sexuels (40\%). Trois cas de figure semblent se dessiner : 1'arrêt de toute activité sexuelle, situation la plus fréquente; continuation d'une activité sexuelle occasionnelle avec homme(s) et femme(s); continuation d'une activité sexuelle avec un partenaire principal.

Pierre, 81 ans, a arrêté l'activité sexuelle suite à la découverte du VIH : «[La contamination] a stoppé la vie sexuelle... je me suis replié sur moi. » Adam, 62 ans : «Dès que j’ai su que j'étais séropositif, makach walou [plus rien]. »Cédric, 64 ans : «Au point de vue sexuel, j'ai tout arrêté. » Tous vivaient seuls au moment du dépistage. Pierre et Cédric n'ont jamais vécu en couple. Adam a été marié, mais avait divorcé bien avant sa contamination. La situation de ces hommes ressemble donc à celle des hommes bisexuels séropositifs de moins de 50 ans du groupe de comparaison : ni vie de couple, ni partenaire sexuel.

Quelques hommes bisexuels continuent l'activité sexuelle avec les deux sexes. Philippe, 54 ans: «[Avez-vous une vie sexuelle actuellement?] Oui. Avec hommes et femmes.» Mamadou, 61 ans : "J'ai toujours une vie sexuelle, heureusement. J'ai des femmes et j'ai des amis aussi. » Pour ces hommes, l'activité sexuelle multiple n'a pas été freinée par la séropositivité. Les deux types de partenaires leur sont nécessaires. Philippe, 54 ans : «J'aime vivre avec une femme. J'aime la complémentarité, j'aime la douceur d'une femme. Un homme c'est un bon copain mais ça peut pas être une relation amoureuse. C'est plus une relation de sexe, s'amuser, on a du sexe comme on ferait de la moto ensemble ou des choses comme ça. » Mamadou, 61 ans : «Les femmes aiment beaucoup l'affection, le charme. [Les hommes] c'est pas la même chose. Si ça va pas, on va avec d'autres. » On retrouve à la fois les malentendus entre certains hommes hétérosexuels et leurs compagnes, et la dissociation entre affectivité et sexualité des hommes homosexuels. 
Parmi les seniors bisexuels, un seul a été contaminé lors d'un rapport extraconjugal, à l'insu de sa conjointe. La découverte du VIH fut alors un choc. Dominique, 62 ans : «Ben, ça a été très dur, au départ... [la bisexualité et la séropositivité] sont venus en même temps, voilà. Je vais pas dire qu'elle m'a pardonné, mais elle a surmonté l'épreuve. » Dominique a arrêté les rapports avec les hommes, mais continue une activité sexuelle avec son épouse.

Les seniors bisexuels ne ressemblent donc pas aux seniors homosexuels avec lesquels ils sont le plus souvent confondus dans la catégorie HSH. S'ils maintiennent une certaine activité sexuelle, c'est surtout grâce à la minorité multipartenaire et multigenre. Pour cette minorité, la découverte de la séropositivité ne constitue pas un frein durable à la vie sexuelle. Pour la majorité, en revanche, elle signifie la fin de l'activité sexuelle et de la vie de couple. Les seniors bisexuels sont sensiblement plus isolés, et psychologiquement plus fragiles, que les seniors homosexuels.

\subsection{Femmes}

La part des femmes déclarant un partenaire sexuel est moins élevée après l'âge de 50 ans (40 \%) qu'avant (60 \%). La vie de couple, elle, semble stable : $40 \%$ avant 50 ans, $42 \%$ après. Contrairement aux seniors homosexuels, les femmes vivent le plus souvent (73\%) en couple sérodiscordant. La contamination par le conjoint est assez exceptionnelle. Seule une femme est dans la situation de la veuve telle que décrite par Desesquelles et al. (2013), c'est-à-dire contaminée par son conjoint décédé depuis.

Une comparaison entre femmes et hommes hétérosexuels, qui sont les partenaires de contamination, montre davantage de complémentarité que de ressemblance. On ne retrouve ni l'effondrement de l'activité sexuelle constaté chez les hommes, ni leur niveau relativement élevé de vie de couple. La différence remonte à la contamination. Celle-ci semble avoir eu lieu le plus souvent entre hommes vivant en couple et femmes vivant seules. Une fois le virus dépisté, les hommes continuent la vie de couple qui devient alors conflictuelle et asexuelle. Les femmes continuent leur vie seule, ou alors - cas de figure minoritaire - se remettent en couple avec un nouveau compagnon, généralement séronégatif. Ces nouveaux couples sont alors tous sexuellement actifs et conjugalement satisfaisants.

La principale différence entre les femmes et les hommes homosexuels concerne la dissociation entre affection et sexualité, dissociation revendiquée et pratiquée par les hommes homosexuels, absente chez les femmes. Toutes les femmes vivant en couple ont des rapports sexuels avec leur conjoint et, inversement, toutes les femmes déclarant un partenaire sexuel 
vivent avec lui. Cela ne signifie pas qu'il n'y ait pas de multipartenariat. Charlotte, 56 ans, adepte du SM, continue les échanges sexuels : «Plus trop.... malheureusement (rires). A part mon mari... quoique, il y a pas très longtemps, il y a quelqu'un qui est venu à la maison. »

Claude, 55 ans, a eu un parcours similaire: "J'ai eu une vie sexuelle anarchique.... j'ai rencontré de la drogue, et je suis partie dans une vie avec les boîtes échangistes... le monde de la nuit quoi.»

Il n'en reste pas moins que la majorité des femmes seniors séropositives n'ont ni partenaire sexuel, ni conjoint. Certaines sont veuves, mais rarement d'un mari séropositif. D'autres se sont séparées. Du point de vue conjugal, elles sont souvent isolées. Mais la plupart des femmes seniors ont des enfants et parfois des petits-enfants. Contrairement aux hommes seniors hétéro- ou bisexuels, eux aussi avec enfants et petits-enfants, les femmes seniors se disent toujours en très bons termes avec leurs enfants.

\section{Discussion}

Les principales limites de notre enquête viennent de sa faible représentativité. L'échantillon est tiré de la population vieillissant avec le VIH et suivie dans les hôpitaux du COREVIH Vallée du Rhône, mais ni le taux de participation ni les motifs de refus n'ont pu être estimés. Ensuite, les deux modes de recueil de témoignages, questionnaires et entretiens, quoique conçus sur le même modèle, ont produit un corpus hétérogène, dont l'analyse n'a pas toujours été aisée. La faiblesse du volet quantitatif n'a pas toujours pu être compensée par la richesse du volet qualitatif.

Néanmoins, l'analyse des mécanismes sociaux propres à chaque catégorie de seniors montre suffisamment de différences entre les catégories et suffisamment d'homogénéité à l'intérieur de celles-ci, pour qu'ils puissent être considérés comme des hypothèses de travail pour de futures recherches.

\section{Conclusion}

Comme attendu, les seniors de notre enquête déclarent peu d'activité sexuelle. Dans toutes les catégories, les sexuellement actifs sont minoritaires. L'écart avec la population générale française est important. A l'âge des seniors de l'enquête (50-59 ou 60-69 ans), les hommes français sont sexuellement actifs pour respectivement $94 \%$ et $89 \%$, les femmes françaises pour $86 \%$ et $66 \%$ (Bajos et al., $2008: 354$ ). Aucune des catégories de seniors de notre 
enquête n'atteint $50 \%$. Les hommes hétérosexuels, contrairement aux résultats de Lovejoy et al. (2008), ne le sont que pour $32 \%$.

Dans l'ensemble, l'activité sexuelle des seniors séropositifs est donc aussi faible que celle des personnes ayant une maladie chronique (Colson, 2016; Levinson, 2008). Mais la ressemblance s'arrête là. En effet, si d'autres maladies chroniques réduisent l'activité sexuelle soit par des dysfonctions physiologiques, soit sous l'effet d'anxiétés ou de dépressions, le VIH a un rapport plus direct avec la sexualité. Il confronte le couple aux pratiques sexuelles passées et futures du couple mais aussi de chaque partenaire. Bien plus que les dysfonctions physiologiques ou psychologiques, c'est la discorde conjugale qui nourrit l'inactivité sexuelle des seniors séropositifs, une discorde dont les mécanismes sociaux s'avèrent très différents pour les différentes catégories de seniors séropositifs.

Les hommes hétérosexuels sont les moins nombreux à avoir des relations sexuels, tout en étant les plus nombreux à vivre en couple (58 \%). Il s'agit en général de couples anciens, qui se sont maintenus après la découverte de la contamination. Aucun n'a maintenu une activité sexuelle. Bien souvent les couples avaient déjà cessé les relations sexuelles. Dans un cas comme dans l'autre, l'homme senior hétérosexuel n'est pas socialement isolé. Il vit en couple, mais en couple conflictuel et le conflit s'étend souvent aux enfants et parfois aux petitsenfants. S'il n'est pas socialement isolé, il l'est bien souvent affectivement.

Les femmes seniors hétérosexuelles ont peu de relations sexuelles et également peu de vie conjugale. Mais quand elles sont en couple - le plus souvent avec un homme séronégatif -, elles ont une activité sexuelle avec leur partenaire et, inversement, quand elles ont une activité sexuelle, elles vivent en couple. Malgré le fait que les partenaires des femmes et hommes hétérosexuels, si partenaire y a, sont le plus souvent séronégatifs, leur situation conjugale est à radicalement différente du point de vue de l'activité sexuelle et de la satisfaction conjugale. L'origine de cette différence tient à la constitution des couples. Presque tous les couples des femmes seniors se sont constitués après la découverte du VIH. Les conjointes des hommes séropositifs n'ont pas été contaminées puisque l'activité sexuelle conjugale fut faible ou inexistante. Les conjoints des femmes séropositives sont séronégatifs, car le statut sérologique de leur compagne était connu dès la constitution du couple.

Plus harmonieuse et plus satisfaisante, la vie de couple des femmes séropositives est néanmoins minoritaire. La majorité des femmes seniors séropositives n'ont pas de partenaire 
sexuel ni conjoint de vie. Souvent contaminées lors de relations courtes non cohabitant, elles ont tendance à se replier sur elles-mêmes et leurs enfants.

Les hommes homosexuels suivent un autre parcours encore. Ils sont assez nombreux à avoir des relations sexuelles et assez nombreux à vivre en couple. Mais les partenaires sexuels ne sont pas toujours les conjoints, ni inversement. Dans la plupart des cas, les couples sont de nouveaux couples, constitués après la « rupture biographique » (Bury, 1982) que constitue la découverte du VIH, et ces nouveaux couples sont séroconcordants. Les couples se disent souvent «ouverts» et les relations sexuelles sont davantage extra- qu'intraconjugales. Cependant, à l'extérieur du domicile, l'âgisme réduit considérablement l'activité sexuelle des seniors homosexuels. Le recul de l'activité sexuelle, à l'intérieur comme à l'extérieur du couple, semble paradoxalement préserver le couple, désormais fondé avant tout sur l'affection. Il s'accompagne même d'une multiplication de la vie de couple cohabitant, dont le taux passe de $32 \%$ avant l'âge de 50 ans à $50 \%$ après.

Enfin, les hommes bisexuels constituent un quatrième cas de figure, très différent encore des trois autres, notamment de celui des hommes homosexuels avec qui ils sont souvent confondus. Ils sont aussi peu nombreux que les femmes à déclarer des relations sexuelles, et beaucoup moins nombreux encore à vivre en couple. Une minorité est sexuellement très active dans des pratiques multipartenaires et multigenres, vivant en couple ou pas. La majorité, toutefois, n'est ni sexuellement actif, ni en couple. Il s'agit probablement des seniors socialement les plus isolés socialement, mais aussi affectivement, puisque les rapports avec les ex-conjointes et avec les enfants issus des couples précédents peuvent être très conflictuels.

Ainsi, si l'activité sexuelle est faible pour tous les seniors vivant avec le VIH, les dynamiques qui la produisent et les conséquences sociales et psychologiques sont très différentes selon que l'on est homme ou femme, homo-, bi- ou hétérosexuel.

L'auteur déclare ne pas avoir de liens d'intérêts.

\section{Références bibliographiques}

Adam P. Bonheur dans le ghetto ou bonheur domestique ? Enquête sur l'évolution des expériences homosexuelles. Actes de la recherche en sciences sociales 1999 ; 128 : 5667. 
ANRS. 8e Séminaire de recherche clinique sur l'infection par le VIH. Paris : ANRS ; 2009.

Bajos N, Bozon M. Enquête sur la sexualité en France. Pratiques, genre et santé. Paris : La Découverte ; 2008.

Banens M, Mendès-Leite R, Talpin J-M, Cuvillier B. Vieillir avec le VIH. Les seniors séropositifs à Lyon et dans la Vallée du Rhône. Lyon : CMW Working Paper ; 2015.

Bury, M. (1982). Chronic illness as biographical disruption. Sociology of health and illness, 4(2), 167-182.

Colson M.-H. Dysfonctions sexuelles de la maladie chronique, l'état des lieux. Première partie : fréquence, impact et gravité. Sexologies 2016, 25 (1): 16-23. doi:10.1016/j.sexol.2016.01.008.

Desesquelles A, Gotman A, Micheau J, Molière E. Etude sur la prise en charge des personnes vieillissantes vivant avec le VIH/SIDA. Paris : Direction générale de la santé ; 2013.

Golub SA, Grov C, Tomassilli J. Sexual behavior among HIN+ older adults. In : Brennan M. et al. (dir). Older adults with HIV : an indepth examination of an emerging population. NY: Nova Science Publications; 2009: 43-50

Groupe SOS, SOS Homophobie, AIDES. Rapport sur le vieillissement des personnes LGBT et des personnes séropositives au VIH. 2013.

Iwuji C, Churchill D, Gilleece Y, Weiss H, Fisher M. Older HIV-Infected Individuals Present Late and Have a Higher Mortality: Brighton, UK Cohort Study. BMC Public Health 2013, 13 (1): 397. doi:10.1186/1471-2458-13-397.

Joyce G, Dana P, Goldman A, Leibowitz A, Yuhua B. A Socioeconomic Profile of Older Adults With HIV. Journal of Health Care for the Poor and Underserved 2005; 16: 19-28.

Kontula O, Haavio-Mannila E. The impact of aging on human sexual activity and sexual desire. J Sex Res 2009, 46 (1) : 46-56.

Le Van C, Ferrand M, Levinson S, L'absence d'activité sexuelle : une singularité plurielle. In : Bajos N, Bozon M. Enquête sur la sexualité en France. Paris : La Découverte ; 2008, p. 333-355.

Lert F, Pialoux G. Prévention et réduction des risques dans les groupes à haut risque vis-à-vis du VIH et des IST. Rapport - Missions RDRs. 2010.

Lert F, Annequin M, Tron L, Aubrière C, Hamelin C, Spire B, Dray-Spira R. Situation socioéconomique des personnes vivant avec le VIH suivies à l'hôpital en France métropolitaine en 2011. Premiers résultats de l'enquête ANRS-VESPA2. Bulletin épidémiologique hebdomadaire 2013 ; 26-27: 293-99.

Levinson S. Les "difficultés” de la function sexuelle: contexts, determinant et significations. In : Bajos N, Bozon M. Enquête sur la sexualité en France. Paris : La Découverte ; 2008, p. 485-508.

Lovejoy T, Heckman T, Sikkema K, Hansen N, Kochman A, Suhr J, Garske J, Johnson C. Patterns and Correlates of Sexual Activity and Condom Use Behavior in Persons 50Plus Years of Age Living with HIV/AIDS. AIDS and Behavior 2008; 12: 943-56.

Morlat, P. Prise en charge médicale des personnes vivant avec le VIH : actualisation $2014 \mathrm{du}$ rapport 2013. Ministère des Affaires sociales, de la Santé et des Droits des femmes, 2014. http://www.sante.gouv.fr/prise-en-charge-medicale-des-personnes-vivant-avecle-vih-actualisation-2014-du-rapport-2013.html. 
Trudel G, Dargis L, Villeneuve L, Cadieux J, Boyer R, Préville M. Fonctionnement conjugal, sexuel et psychologique des couples aînés vivant à domicile: les résultats d'une enquête nationale avec méthodologie longitudinale (première partie). Sexologies, 2013, 22 (4): 176-83. doi:10.1016/j.sexol.2013.03.004.

- 2014. Fonctionnement conjugal, sexuel et psychologique des couples aînés vivant à domicile : les résultats d'une enquête nationale avec méthodologie longitudinale (deuxième partie). Sexologies, 2014, 23 (2): 52-68. doi:10.1016/j.sexol.2013.03.006.

UNAIDS. HIV and aging. A special supplement to the UNAIDS report on the global AIDS epidemic 2013. Genève: UN.

Wallach I, Ducandas X, Martel M, Trottier B, Thomas R. Le VIH et le vieillissement au Québec. Une recherche qualitative sur les expériences, les difficultés et les besoins des personnes vivant avec le VIH de 50 ans et plus. Ministère de la Famille et des Aînés 2013.

Wallach I. «Je suis heureux d'avoir l'âge que j'ai »: la résilience des hommes gais âgés vivant avec le VIH au Québec. Canadian Journal of Community Mental Health 2011; 30: $157-71$.

Widmer ED, Ammar N. Désir sexuel et styles d'interactions conjugales. Sexologies 2013, 22 (4): 150-58. doi:10.1016/j.sexol.2013.05.003. 